\title{
Nucleon Polarisabilities and Compton Scattering on ${ }^{3} \mathrm{He}$
}

\section{Harald W. Grießhammer*}

Institute for Nuclear Studies, Department of Physics, George Washington University,

Washington DC 20052, USA; E-mail: hgrie@gwu . edu

\section{Judith A. McGovern}

School of Physics and Astronomy, The University of Manchester, Manchester M13 9PL, UK

\section{Daniel R. Phillips}

Department of Physics and Astronomy, Institute of Nuclear and Particle Physics,

Ohio University, Athens $\mathrm{OH} 45701, \mathrm{USA}$

\footnotetext{
This executive summary of recent theory progress in Compton scattering off ${ }^{3} \mathrm{He}$ focuses on determining neutron polarisabilities; see ref. [1] and references therein for details and a better bibliography.
}

The 9th International workshop on Chiral Dynamics 17-21 September 2018

Durham, NC, USA

${ }^{*}$ Speaker. 


\section{Setting the Stage}

Low-energy Compton scattering $\gamma X \rightarrow \gamma X$ probes a target's internal degrees of freedom in the electric and magnetic fields of a real, external photon. These fields induce radiation multipoles by displacing the target constituents. The angular and energy dependence of the emitted radiation encodes information from the symmetries and strengths which govern the interactions of the constituents with each other and with photons.

A series of plenaries and parallel talks discussed the large-scale international effort, gains and goals of a new generation of high-precision facilities to understand low-energy Nuclear Physics by extracting nucleon polarisabilities from Compton scattering experiments [2]. Some highlight the importance of electromagnetic polarisabilities in other contexts; others showed that determining them by experiments takes years of planning, execution and analysis - and commensurate theory support. We refer to all these contributions for motivation and context, and concentrate on theory progress for one target nucleus, ${ }^{3} \mathrm{He}$, summarising the essentials of a recent publication [1]. A somewhat more elaborate exposition can be found in a plenary talk of this conference [3].

After subtracting the "Born contributions" (known from one-photon data like form factors), its multipoles parametrise the stiffness of a nucleon $N$ (spin $\frac{\vec{\sigma}}{2}$ ) against transitions of multipolarity $X l \rightarrow Y l^{\prime}$ at frequency $\omega\left(l^{\prime}=l \pm\{0 ; 1\} ; X, Y=E, M ; T_{i j}=\frac{1}{2}\left(\partial_{i} T_{j}+\partial_{j} T_{i}\right) ; T=E, B\right)$ :

$$
\begin{aligned}
2 \pi N^{\dagger}[ & \alpha_{E 1}(\omega) \vec{E}^{2}+\beta_{M 1}(\omega) \vec{B}^{2}+\gamma_{E 1 E 1}(\omega) \vec{\sigma} \cdot(\vec{E} \times \dot{\vec{E}})+\gamma_{M 1 M 1}(\omega) \vec{\sigma} \cdot(\vec{B} \times \dot{\vec{B}}) \\
& \left.-2 \gamma_{M 1 E 2}(\omega) \sigma^{i} B^{j} E_{i j}+2 \gamma_{E 1 M 2}(\omega) \sigma^{i} E^{j} B_{i j}+\text { (higher multipoles) }\right] N .
\end{aligned}
$$

Six two-photon response functions suffice up to about $400 \mathrm{MeV}$ : two scalar polarisabilities $\alpha_{E 1}(\omega)$ and $\beta_{M 1}(\omega)$ for electric and magnetic dipole transitions; and the four dipole spin-polarisabilities $\gamma_{E 1 E 1}(\omega), \gamma_{M 1 M 1}(\omega), \gamma_{E 1 M 2}(\omega), \gamma_{M 1 E 2}(\omega)$. These test the nucleon-spin structure and complement information from Jefferson Lab's spin programme. Intuitively, the electromagnetic field of the spin degrees causes bi-refringence in the nucleon, like in the classical Faraday-effect.

Since they are related to the (real and virtual) excitation spectrum of the target, they probe the two-photon response of a nucleon, complementing the information available in the one-photon response (e.g. in form factors). Differences between proton and neutron values stem from isospinbreaking interactions, exploring the interplay between chiral symmetry as well as the pattern of its breaking, and short-distance Physics.

The static values, $\alpha_{E 1} \equiv \alpha_{E 1}(\omega=0)$ etc., are often just called "the" polarisabilities and condense the rich information on the pion cloud, on the $\Delta(1232)$ excitation, and on the interplay between chiral symmetry breaking and short-distance interactions. These fundamental quantities provide stringent tests for theoretical descriptions of hadron structure. Moreover, they are ingredients to the neutron-proton mass difference, the proton charge-radius puzzle, and the Lamb shift of muonic hydrogen. To extract them, one must reliably extrapolate from data to $\omega=0$.

Since pure neutron targets are unfeasible, nuclear binding and meson-exchange effects must also be subtracted with reliable theory uncertainties. Fortunately, Chiral Effective Field Theory $(\chi \mathrm{EFT})$ provides model-independent estimates of higher-order corrections and encodes the correct low-energy dynamics of QCD. For few-nucleon systems, it consistently incorporates hadronic and nuclear currents, rescattering effects and wave functions. The photon's interaction with the charged pion-exchange between nucleons also probes few-nucleon binding. Even if scattering on a free 


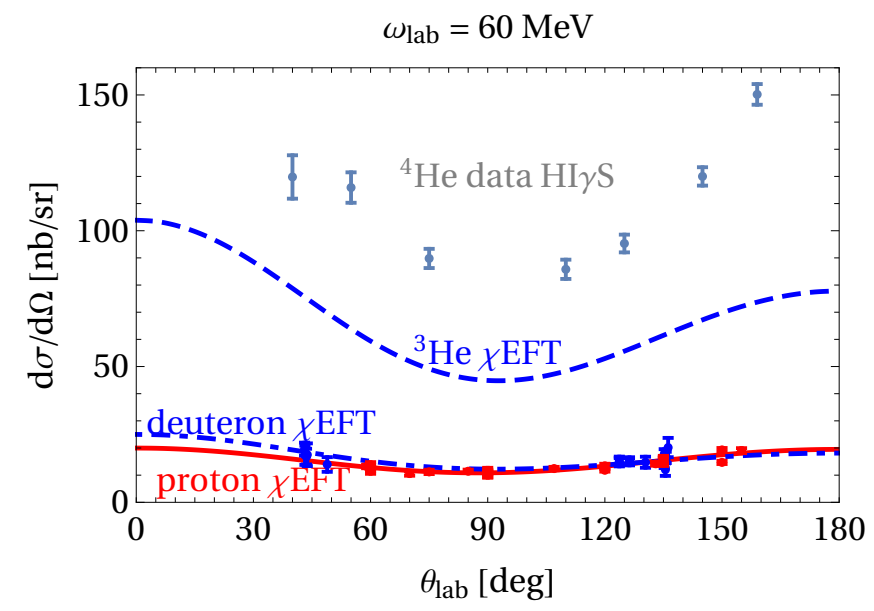

Figure 1: Differential cross section of Compton scattering at $\omega_{\mathrm{lab}}=60 \mathrm{MeV}$ for the neutron (zero), proton (red solid), deuteron (blue dot-dashed), ${ }^{3} \mathrm{He}$ (blue dashed) and ${ }^{4} \mathrm{He}$ (green dotted; data). Data are shown as available for the proton, deuteron and ${ }^{4} \mathrm{He}$; no theory for ${ }^{4} \mathrm{He}$ is available yet. The lines are $\chi \mathrm{EFT}$ predictions, all using the same unified framework.
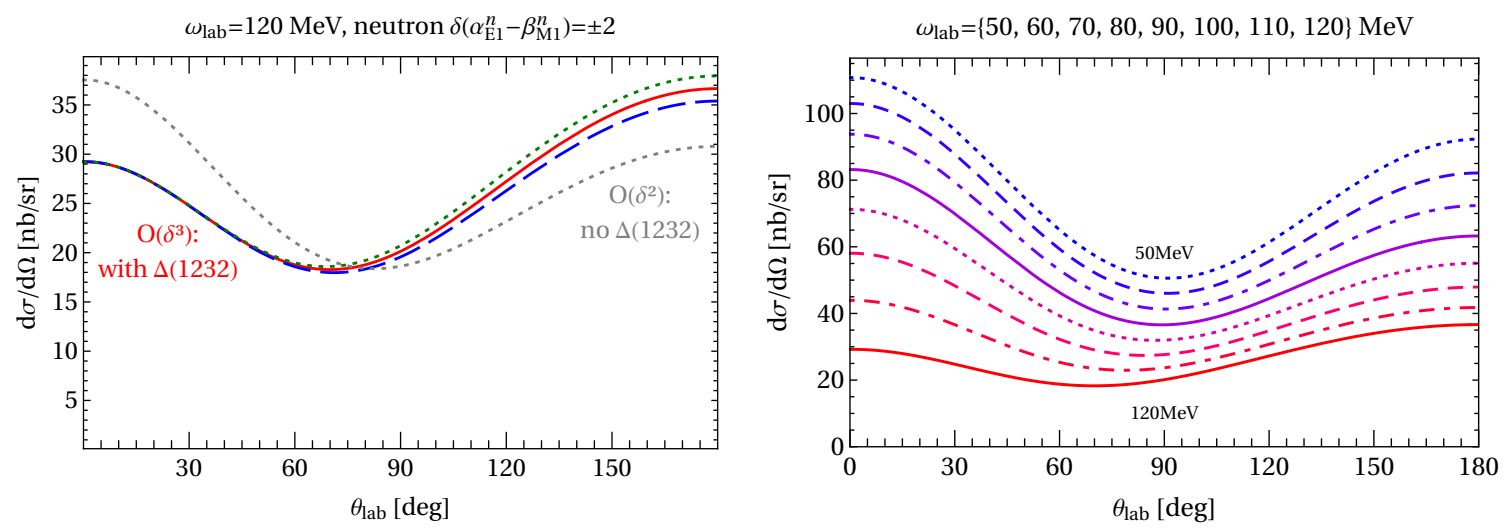

Figure 2: Differential cross section of ${ }^{3} \mathrm{He}$ Compton scattering at $\omega_{\mathrm{lab}}=120 \mathrm{MeV}$, adapted from [1]. Left: at $\mathscr{O}\left(e^{2} \delta^{2}\right)$ [no Delta] and $\mathscr{O}\left(e^{2} \delta^{3}\right)$ [with Delta], and sensitivity to the neutron's scalar polarisabilities. Right: Energy dependence.

neutron were feasible, cross sections and signals for coherent scattering from nuclei are markedly larger, as fig. 1 demonstrates.

\section{Elastic Compton scattering from ${ }^{3} \mathrm{He}$}

This is is a promising means to access neutron polarisabilities. In ref. [4] and subsequent publications, Shukla et al. showed that the differential cross section between 50 and $120 \mathrm{MeV}$ is sensitive to the electric and magnetic dipole polarisabilities of the neutron, $\alpha_{E 1}^{(\mathrm{n})}$ and $\beta_{M 1}^{(\mathrm{n})}$, and that scattering on polarised ${ }^{3} \mathrm{He}$ provides good sensitivity to the neutron spin polarisabilities. These findings triggered several approved proposals at MAMI and HI $\gamma \mathrm{S}$ to exploit this opportunity to extract neutron polarisabilities from elastic $\gamma^{3} \mathrm{He}$ scattering. 
We recently extended these $\chi$ EFT predictions by one order to $\mathrm{N}^{3} \mathrm{LO}\left[\mathscr{O}\left(e^{2} \delta^{3}\right)\right]$ by adding a dynamical Delta degree of freedom, and provided results for photon lab energies between 50 and $120 \mathrm{MeV}$ for the differential cross section, for the beam asymmetry $\Sigma^{\text {lin }}=\Sigma_{3}$, and for the two double asymmetries with circularly polarised photons and transversely or longitudinally polarised targets, $T_{11}^{\text {circ }}=-\sqrt{2} \Sigma_{2 x}$ and $T_{10}^{\text {circ }}=\Sigma_{2 z}$. These are the only non-zero observables below pionproduction threshold in our formulation. We also found that the pioneering results were obtained from a computer code which contained mistakes, triggering an erratum to ref. [4].

We employ essentially the same $\gamma N$ and $\gamma N N$ amplitudes as in nucleon and deuteron Compton scattering. At such energies, the complete photonuclear operator at $\mathrm{N}^{3} \mathrm{LO}\left[\mathscr{O}\left(e^{2} \delta^{3}\right)\right]$ is: the Thomson and other minimal-substitution terms; magnetic-moment couplings; dynamical singlenucleon effects such as virtual pion loops and the Delta excitation; and couplings of photons to the charged-pion exchange. All terms are evaluated with ${ }^{3} \mathrm{He}$ wave functions found from the same $\chi$ EFT expansion.

The kinematics of a differential cross section with definite beam and target polarisations (and no detection of the final-state polarisations) is summarised in fig. 3. It can be written as

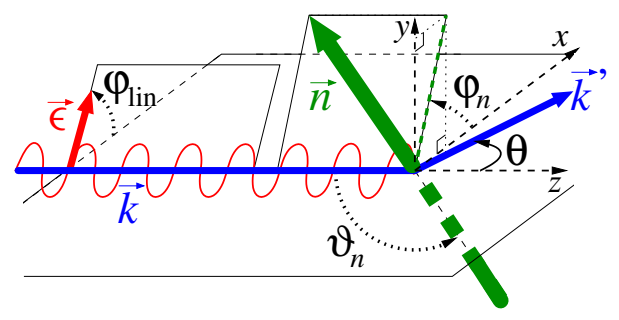

Figure 3: (Colour on-line) Kinematics of ${ }^{3} \mathrm{He}$ Compton scattering.

$$
\begin{aligned}
\left.\frac{\mathrm{d} \sigma}{\mathrm{d} \Omega}\right|_{\text {unpol }}[ & 1+\xi_{3} \Sigma_{3}(\omega, \theta)+P^{\left({ }^{3} \mathrm{He}\right)} n_{y} \Sigma_{y}(\omega, \theta) \\
& +P^{\left({ }^{3} \mathrm{He}\right)} \xi_{1}\left(n_{x} \Sigma_{1 x}(\omega, \theta)+n_{z} \Sigma_{1 z}(\omega, \theta)\right) \\
& \left.+P^{\left({ }^{3} \mathrm{He}\right)} \xi_{2}\left(n_{x} \Sigma_{2 x}(\omega, \theta)+n_{z} \Sigma_{2 z}(\omega, \theta)\right)+P^{\left({ }^{3} \mathrm{He}\right)} \xi_{3} n_{y} \Sigma_{3 y}(\omega, \theta)\right],
\end{aligned}
$$

where $\xi_{i}$ are the Stokes parameters of photon polarisation and the target polarisation of degree $P^{\left({ }^{3} \mathrm{He}\right)}$ is pointing along the normal vector $\vec{n}$. It is fully parametrised by 8 linearly independent functions listed below. Here, $\mathrm{d} \sigma$ is shorthand for $\mathrm{d} \sigma / \mathrm{d} \Omega$; superscripts refer to photon polarisations (" $\|$ " for polarisation in the scattering plane, " $\perp$ " for perpendicular to it); subscripts to target polarisations; and the absence of either means unpolarised. The observables are:

- 1 differential cross section $\left.\frac{\mathrm{d} \sigma}{\mathrm{d} \Omega}\right|_{\text {unpol }}$ of unpolarised photons on an unpolarised target.

- 1 beam asymmetry of a linearly polarised beam on an unpolarised target:

$$
\Sigma^{\operatorname{lin}}=\Sigma_{3}=\frac{\mathrm{d} \sigma^{\|}-\mathrm{d} \sigma^{\perp}}{\mathrm{d} \sigma \|+\mathrm{d} \sigma^{\perp}} .
$$


- 1 vector target asymmetry for a target polarised out of the scattering plane along the $\pm y$ direction and an unpolarised beam:

$$
T_{11}=-\sqrt{2} \Sigma_{y}=-\sqrt{2} \frac{\mathrm{d} \sigma_{y}-\mathrm{d} \sigma_{-y}}{\mathrm{~d} \sigma_{y}+\mathrm{d} \sigma_{-y}} .
$$

- 2 double asymmetries of right-/left-circularly-polarised photons on a target polarised along the $\pm x$ or $\pm z$ directions:

$$
T_{11}^{\mathrm{circ}}=-\sqrt{2} \Sigma_{2 x}=-\sqrt{2} \frac{\mathrm{d} \sigma_{x}^{R}-\mathrm{d} \sigma_{x}^{L}}{\mathrm{~d} \sigma_{x}^{R}+\mathrm{d} \sigma_{x}^{L}}, T_{10}^{\mathrm{circ}}=\Sigma_{2 z}=\frac{\mathrm{d} \sigma_{z}^{R}-\mathrm{d} \sigma_{z}^{L}}{\mathrm{~d} \sigma_{z}^{R}+\mathrm{d} \sigma_{z}^{L}}
$$

- 3 double asymmetries of linearly-polarised photons on a vector-polarised target:

$$
\begin{aligned}
T_{1 \pm 1}^{\operatorname{lin}=} & \left( \pm \Sigma_{1 x}-\Sigma_{3 y}\right) / \sqrt{2} \\
& \text { with } \Sigma_{1 x}=\frac{\mathrm{d} \sigma_{x}^{\pi / 4}-\mathrm{d} \sigma_{x}^{-\pi / 4}}{\mathrm{~d} \sigma_{x}^{\pi / 4}+\mathrm{d} \sigma_{x}^{-\pi / 4}}, \Sigma_{3 y}=\frac{\left(\mathrm{d} \sigma_{y}^{\|}-\mathrm{d} \sigma_{y}^{\perp}\right)-\left(\mathrm{d} \sigma_{-y}^{\|}-\mathrm{d} \sigma_{-y}^{\perp}\right)}{\mathrm{d} \sigma_{y}^{\|}+\mathrm{d} \sigma_{y}^{\perp}+\mathrm{d} \sigma_{-y}^{\|}+\mathrm{d} \sigma_{-y}^{\perp}}, \\
T_{10}^{\operatorname{lin}}= & -\Sigma_{1 z}=-\frac{\mathrm{d} \sigma_{z}^{\pi / 4}-\mathrm{d} \sigma_{z}^{-\pi / 4}}{\mathrm{~d} \sigma_{z}^{\pi / 4}+\mathrm{d} \sigma_{z}^{-\pi / 4}} .
\end{aligned}
$$

The decomposition of eq. (2.1) holds in any frame, but the functions are frame-dependent. Of these functions, only the cross section, beam asymmetry and circular-polarisation asymmetries $\Sigma_{2 x / z}$ are appreciably nonzero. Here, we also list the observables in the multipole decomposition, $T_{l m}, T_{l m}^{\text {circ }}$ and $T_{l m}^{\mathrm{lin}}$, with $(l m)$ the target state; see ref. [1] for details.

\section{Results}

The dynamical Delta effects do not enter at low energies, but they are obvious in all observables for $\omega_{\text {lab }} \gtrsim 100 \mathrm{MeV}$; see fig. 2. They markedly invert the fore-aft asymmetry of the cross section and increase the magnitude of double asymmetries and their sensitivity to spin polarisabilities, echoing similar findings for the deuteron. The chiral expansion converges in this energy range quite well. The dependence on the choice of the ${ }^{3} \mathrm{He}$ wave function is small and can usually be distinguished from the effects of polarisabilities by a different angular dependence.

We found that $\alpha_{E 1}^{(\mathrm{n})}-\beta_{M 1}^{(\mathrm{n})}$ can be extracted from the cross section. In addition, $T_{11}^{\text {circ }}=-\sqrt{2} \Sigma_{2 x}$ has a non-degenerate sensitivity to $\gamma_{M 1 M 1}^{(\mathrm{n})}$ around $90^{\circ}$; see fig. 5; and $T_{10}^{\text {circ }}=\Sigma_{2 z}$ to $\gamma_{E 1 E 1}^{(\mathrm{n})}$ and $\gamma_{E 1 M 2}^{(\mathrm{n})}$; see fig. 6 . Ono the other hand, the beam asymmetry $\Sigma^{\text {lin }}=\Sigma_{3}$ is dominated by the single-nucleon Thomson term and not very useful to directly determine polarisabilities. Ultimately, the most accurate polarisabilities will be inferred from data of all four observables. For the spin polarisabilities, data at $\omega_{\text {lab }} \gtrsim 100 \mathrm{MeV}$ will be crucial. That follows both by the sensitivity of asymmetries to polarisabilities, and by the overall size of the asymmetry, which enters in rate estimates; see fig. 4.

This exploration is part of an ongoing dialogue with our experimental colleagues on the best kinematics and observables to extract neutron polarisabilities. Results are available as interactive Mathematica notebook from hgrie@gwu.edu, including cross sections, rates and asymmetries when the scalar and spin polarisabilities are varied (including sum rule constraints). Results are 

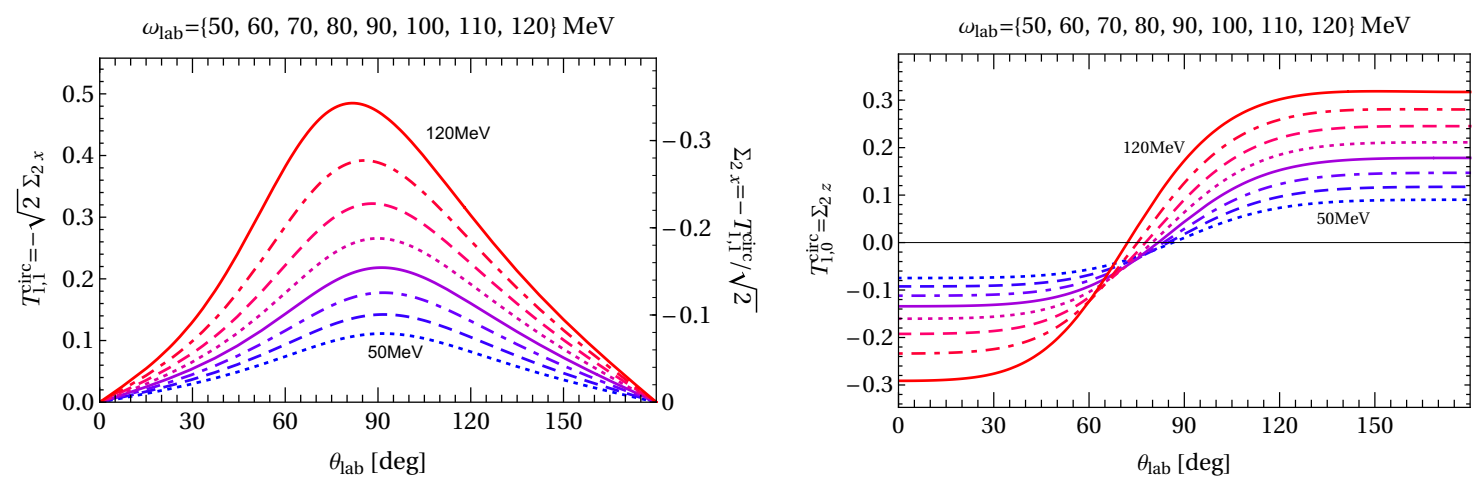

Figure 4: Dependence of the double asymmetries $T_{11}^{\mathrm{circ}}=-\sqrt{2} \Sigma_{2 x}$ (left) and $T_{10}^{\mathrm{circ}}=\Sigma_{2 z}$ (right) at photon energies $\omega_{\text {lab }}=50 \mathrm{MeV}$ to $120 \mathrm{MeV}$ in $10 \mathrm{MeV}$ steps, adapted from [1].
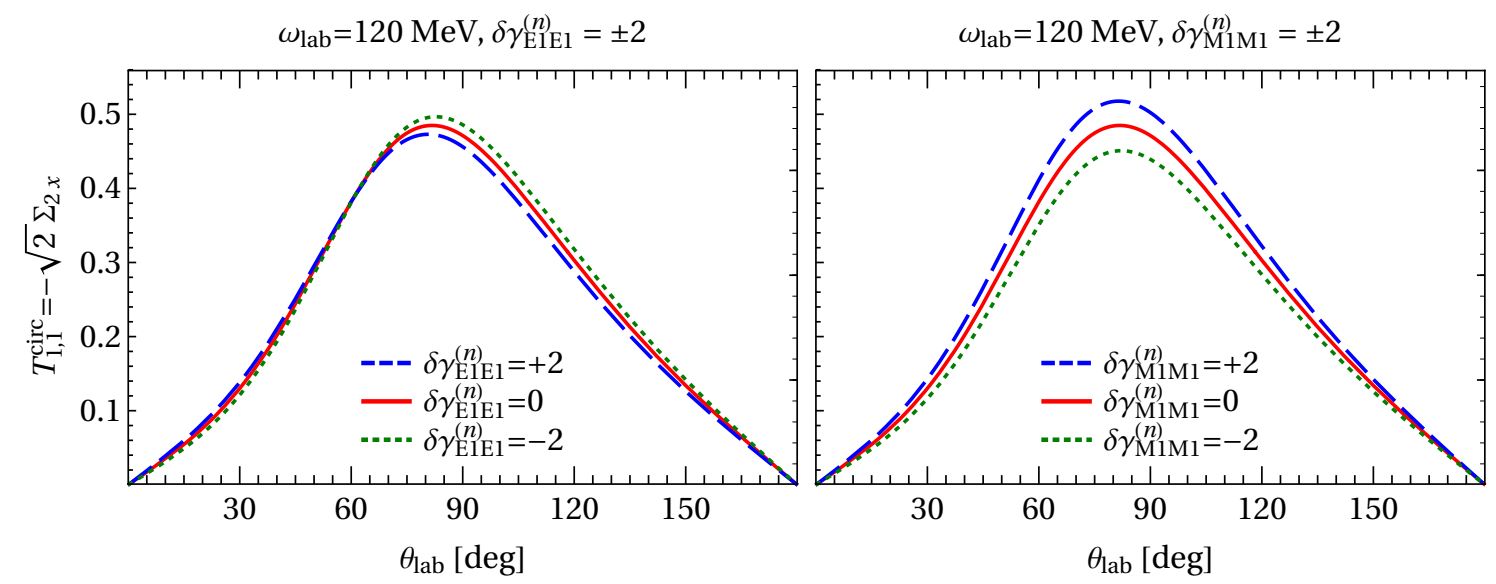

Figure 5: Sensitivity of $\Sigma_{2 x}$ (circularyly polarised beam on transversally polarised target) to varying the two spin neutron polarisabilities with the biggest impact about their central values by \pm 2 units, at $\omega_{\text {lab }}=$ $120 \mathrm{MeV}$, adapted from [1].

quite robust. Varying the single-nucleon amplitudes of complementary approaches like dispersion relations will lead to sensitivities which are hardly discernible from ours. Once data exist, a polarisability extraction will of course need to address residual theory uncertainties with more diligence; see e.g. ref. [5].

\section{Nuclear Binding}

$\chi$ EFT also quantifies the angle- and energy-dependent corrective to the naïve ${ }^{3} \mathrm{He}$ picture as the sum of two protons with antiparallel spins and one neutron. Sensitivity to the scalar polarisabilities enters indeed approximately via $2 \alpha_{E 1}^{(\mathrm{p})}+\alpha_{E 1}^{(\mathrm{n})}$ and $2 \beta_{M 1}^{(\mathrm{p})}+\beta_{M 1}^{(\mathrm{n})}$, and the double-asymmetries are 10to-20 times more sensitive to the spin polarisabilities of the neutron than of the proton. However, fig. 7 confirms that there is no energy where polarised ${ }^{3} \mathrm{He}$ simply acts as a "free neutron-spin target". The sensitivities $\Sigma_{2 x}$ and $\Sigma_{2 z}$ to neutron spin polarisabilities closely mimic those of freeneutron observables. But their magnitudes do not. 


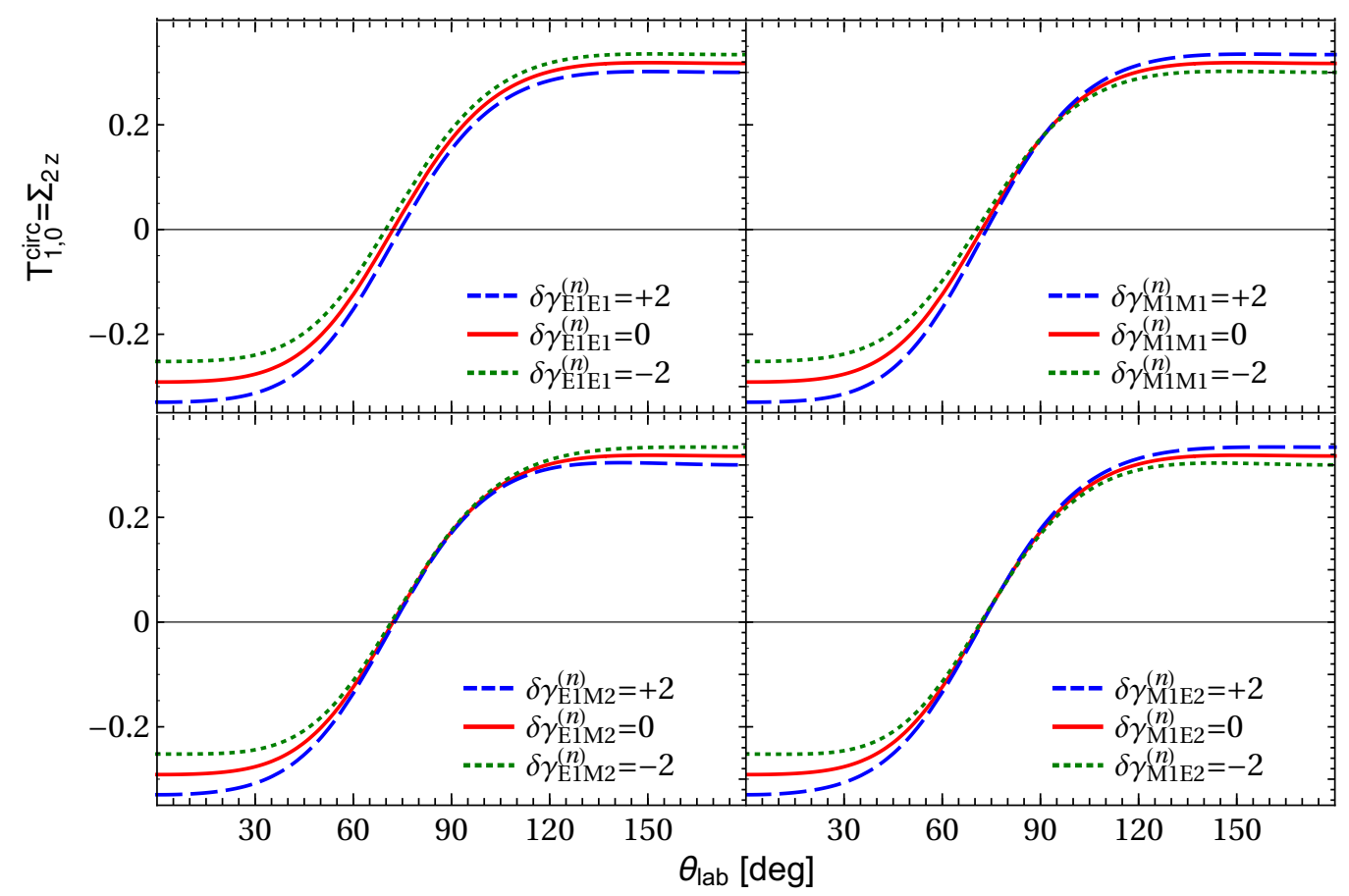

Figure 6: (Colour on-line) Sensitivity of $T_{10}^{\text {circ }}=\Sigma_{2 z}$ (circularyly polarised beam on longitudinally polarised target) to varying the spin polarisabilities of the neutron about their central values by \pm 2 units, at $\omega_{\text {lab }}=$ $120 \mathrm{MeV}$, adapted from [1].
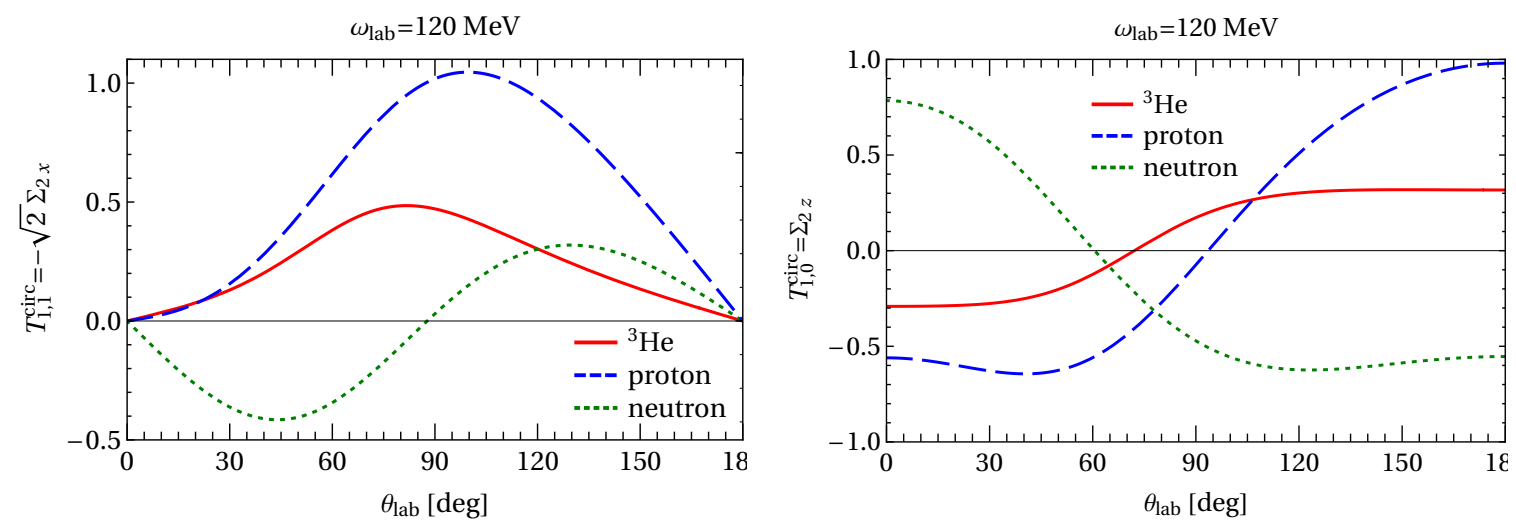

Figure 7: Double asymmetries with circularly polarised beam and transversely (left) or longitudinally (right) polarised target, for proton, neutron and ${ }^{3} \mathrm{He}$, adapted from [1].

Treating the process in impulse approximation would thus omit a key mechanism: charged pion-exchange currents. Their interference with the targeted neutron-structure effects is large, and neglecting them severely distorts extractions of nucleon polarisabilities. The $\chi \mathrm{EFT}$ expansion provides quantitative predictions of the two-body currents, with reliable theory uncertainties. Detailed checks of the convergence of the expansion for exchange currents and for the other pieces of the ${ }^{3} \mathrm{He}$-Compton amplitude by performing a $\mathrm{N}^{4} \mathrm{LO}\left[\mathscr{O}\left(e^{2} \delta^{4}\right)\right]$ calculation and extending the applicable energy range are under way. They will allow for even more accurate extractions of 
polarisabilities from upcoming data.

\section{Acknowledgements}

We are grateful to our co-authors of ref. [1]. All insight is theirs, all mistakes HWG's. This work was supported in part by the US Department of Energy under contracts DE-SC0015393 (HWG) and DE-FG02-93ER-40756 (DRP), and by UK Science and Technology Facilities Council grants ST/L005794/1 and ST/P004423/1 (JMcG).

\section{References}

[1] Margaryan, A. et al.: Eur. Phys. J. A54, 125 (2018) 125 doi:10.1140/epja/i2018-12554-X [arXiv:1804.00956 [nucl-th]].

[2] See the contributions to these proceedings by D. Hornidge, E. Downie, A. Alexandru, Ph. Martel, M. Sikora, L. Myers, R. Miskimen, C. Howell, M. Hoferichter, A. Walker-Loud, R. Young and V. Lensky.

[3] Grießhammer H., McGovern J. A. and Phillips D. R., these proceedings.

[4] Choudhury, D. et al.: Phys. Rev. Lett. 98, 232303 (2007) doi:10.1103/PhysRevLett.120.249901 [nucl-th/0701078]; erratum ibid. 120, 249901 (2018) doi:10.1103/PhysRevLett.98.232303 [arXiv:1804.01206 [nucl-th]].

[5] Grießhammer, H. W. et al: Eur. Phys. J. A52, 139 (2016) doi:10.1140/epja/i2016-16139-5 [arXiv:1511.01952 [nucl-th]]. 